\title{
Modeling the Dependence of Losses of a Financial Portfolio Using Nested Archimedean Copulas
}

\author{
Wendkouni Yaméogo' and Diakarya Barro (iD) ${ }^{2}$ \\ ${ }^{1}$ LANIBIO, Université Joseph Ki-Zerbo, Ouagadougou, Burkina Faso \\ ${ }^{2}$ Université Thomas Sankara, Ouagadougou, Burkina Faso \\ Correspondence should be addressed to Diakarya Barro; dbarro2@gmail.com
}

Received 29 April 2021; Accepted 15 June 2021; Published 15 July 2021

Academic Editor: Niansheng Tang

Copyright (C) 2021 Yaméogo Wendkouni and Diakarya Barro. This is an open access article distributed under the Creative Commons Attribution License, which permits unrestricted use, distribution, and reproduction in any medium, provided the original work is properly cited.

In financial analysis, stochastic models are more and more used to estimate potential outcomes in a risky framework. This paper proposes an approach of modeling the dependence of losses on securities, and the potential loss of the portfolio is divided into sectors each including two subsectors. The Weibull model is used to describe the stochastic behavior of the default time while a nested class of Archimedean copulas at three levels is used to model the maximum of the value at risk of the portfolio.

\section{Introduction}

Stochastic models are more and more used in financial analysis, and they allow institutions to include uncertainties in their estimates, which account for situations where outcomes are risky. In particular, the copula functions are used to capture the dependence of the price movement of financial instruments. The word "copulas" was first introduced by Nelsen [1] in a pioneering work, under the wellknown theorem which bears his name and which clarifies that for any $n$-dimensional distribution $F=\left(F_{1}, \ldots, F_{n}\right)$ with continuous marginal, there exists a unique copula $C_{F}$ mapping $\mathbb{R}^{n}$ to $[0,1]^{n}$ such that $\left(x_{1}, \ldots, x_{n}\right)^{T} \longrightarrow$ $\left(F_{1}\left(x_{1}\right), \ldots, F_{n}\left(x_{n}\right)\right)^{T}$ with

$$
F\left(x_{1}, \ldots, x_{n}\right)=C_{F}\left(F_{1}\left(x_{1}\right) ; \ldots ; F_{n}\left(x_{n}\right)\right) .
$$

Archimedean copulas form a family of copulas used in the construction of multivariate distributions involving onedimensional generator functions.

Well defined by Savu and Trede [2], the hierarchical Archimedean copulas (HACs) offer more flexibility than the classic Archimedean family. In financial modeling, the HACs allow, at the first level, the modeling of the joint dependence of firms including similar sectors. At the second level, the Archimedean copulas, subcopulas of the inners, can beused to combine the sectors. HACs therefore make it possible to model the dependence between a large number of assets in a flexible and intuitive way. Particularly, Gumbel copula, which belongs both to the Archimedean class and the extremal one, is a suitable model used in financial applications (according to Hofert [3]), and it is easy to simulate in hierarchical version (according to McNeil [4]).

The HACs are used in the management of default risk. This default is triggered by a formal credit specified in the contract. This event can be the bankruptcy of the company, a default, or the restructuring of its debt. For the evaluation of these, products derived from credits of intensity models are used. The existing intensity models are constant intensity $\left(\lambda_{t}=\lambda\right)$ by pieces such as

$$
\lambda_{t}=\sum_{k=1}^{q} \lambda_{k} 1_{\left\{T_{k-1}<t \leq T_{k}\right\}} .
$$

In this case, the default time follows the exponential distribution with parameter $\lambda$ intensity governed by an extended CIR process. In the domain of quantitative finance, large portfolio managersneed stochastics models 
whichcombine both nested Archimedean copulas and extreme valueslaws.

In our study, we consider the valuation of credit derivatives that the default intensity also called the hazard rate is given by

$$
h_{\theta}(x, k, \lambda)=\frac{k}{\lambda}\left(\frac{x-\theta}{\lambda}\right)^{k-1}
$$

that is, the default time follows Weibull's law (with $\lambda>0$ and $k \geq 1$ ), whose law covers both a whole family of law.

The major contribution of this paper is the study of the dependence of losses of securities in a large portfolio and the maximum of values at risk of the same portfolio. We show that thhe default time of eachasset follows theWeibull law by using a partially nested Archimedean copula at three levels. The rest of our paper is organized as follows. Section 2 is devoted to the preliminaries of the study where the main properties of both the classical and nested Archimedean copulas and an overview of Weibull negative distribution are given. In Section 3, we study the portfolio losses and default time modeling while Section 4 deals with the nested Archimedean copulas and losses. In Section 5, sampling the hightest values at risk is clarified.

$$
C\left(u_{1}, \ldots, u_{n}\right)=\left\{\varphi\left[\varphi^{-1}\left(u_{1}\right)+\cdots+\varphi^{-1}\left(u_{n}\right)\right], \quad u_{i} \in[0,1]^{n} \text { since } \sum_{i=1}^{n} \varphi\left(u_{i}\right) \leq \varphi(0), 0,\right. \text { else }
$$

where the generalized inverse of the Archimedean generator is given by $\varphi^{-1}(y)=\inf \{t \in[0,1]: \varphi(t) \leq y\}$. The generator must be chosen from class $C^{2}$ (a $C^{2}$ function has both a continuous first derivative and a continuous second derivative) so that $\varphi(1)=0, \varphi^{\prime}(u) \leq 0$ et $\varphi^{\prime \prime}(u)>0$.

Even if Archimedean copulas simulate tails of distributions, their use becomes restrictive for copulas of high-dimensional analysis. Moreover, copula depends on only one parameter which applies to all the steps. The use of hierarchical copulas allows to bypass the restrictions caused by Archimedean copulas. There are partially nested Archimedean copulas and fully nested Archimedean copulas. Here, we use partially nested Archimedean copulas. For example, by coupling margins of the extreme value theory (EVT) with the multivariate Gumbel copula, we obtain the distribution of multidimensional extreme value according to Sklar theorem.

$$
G\left(x_{1}, \ldots, x_{n}\right)=\exp \left(-\left[\sum_{i=1}^{n}\left[-\ln G^{*}\left(x_{i}\right)\right]^{\theta}\right]^{(1 / \theta)}\right)
$$

\section{Materials and Methods}

In this section, we give important definitions and useful properties on Archimedean copulas of extreme values and the value at risk. These results are necessary for our approach. We refer the readers to Schonbucher [5], Capéraà et al. [6], and Cherubini et al. [7] for the applications of Archimedean copulas to different degrees of quantitative finance, Savu and Trede [2] or Hofert and Scherer [8] for hierarchical Archimedean copulas, Veysseyre [9] for details on using Weibull law, and Tyrrell Rockafellar and Uryasev [10] and Roncalli [11] for the value at risk.

2.1. An Overview of Archimedean and Extremal Copulas. Archimedean copulas play an important role in practical applications.

Definition 1. An $n$-dimensional copula $C$ is an Archimedean one if there exists a continuous, strictly decreasing, and convex function $\varphi:[0,1] \longrightarrow[0,+\infty]$, completely monotone on $[0 ; \infty]$, and $(n-2)$ times derivativable such as

$$
(-1)^{k}\left(\varphi^{-1}\right)^{(k)}(t) \geq 0, \quad \text { for all } k=1,2, \ldots, n-2,
$$

called the Archimedean generator function of $C$, such that, for all $\left(u_{1}, \ldots, u_{n}\right) \in[0,1]^{n}$,

where $G^{*}$ is a suitable normalized limit of law of maxima such that

$$
G^{*}\left(x_{i}\right)=\lim _{n \longrightarrow+\infty} P\left[\frac{M_{n-b_{n}}}{a_{n}} \leq x_{i}\right],
$$

with $\left(a_{n}\right)>0$ and $\left(b_{n}\right) \in \mathbb{R}$ being normalizing coefficients and $M_{n}$ being the statistic of maxima. An extreme value copula verifies the following max-stability relation:

$$
C\left(u_{1}^{k}, \ldots, u_{n}^{k}\right)=C^{k}\left(u_{1}, \ldots, u_{n}\right)
$$

for all $u_{i} \in[0,1]$ for $i=1, \ldots, n$.

2.2. An Overview of Weibull Negative Distribution. In stochastic analysis, the Weibull law is a continuous probabilitydistribution within the extreme values family as well as the Gumbel and the Frechet laws. With three parameters (for $x>0$ ), this law is characterized as follows: 


$$
\begin{cases}f_{\theta}(x ; k ; \lambda)=\frac{k}{\lambda}\left(\frac{x-\theta}{\lambda}\right)^{k-1} \exp \left\{-\left(\frac{x-\theta^{k}}{\lambda}\right)\right\}, & \text { the density function, } \\ F_{\theta}(x ; k ; \lambda)=1-\exp \left\{-\left(\frac{x-\theta^{k}}{\lambda}\right)\right\}, & \text { the distribution function, } \\ S_{\theta}(x ; k ; \lambda)=\exp \left(-\frac{x-\theta^{k}}{\lambda}\right), & \text { the survival distribution function, }\end{cases}
$$

where $\theta=\min (x), k>0$ is the shape parameter, and $\lambda>0$ is the scale parameter.

We have below graphical representations of the functions of density and distribution of Weibull law for same values of parameters. Figure lis taken from [12].

One of the uses of Weibull distribution is the extreme value theory where the asymptotic behavior of distributions, mainly for component-wise maxima of laws, is appropriately normalized. Particularly, in one-dimensional extreme framework, the well-known Fisher-Tippett-Gnedenko theorem [13] shows that the three following distributions can characterize these asymptotic behaviors:

$$
\begin{aligned}
\Lambda(x) & =\exp \{-\exp (-x)\} ; \infty<x<\infty, \text { Gumbel distribution, } \\
\Phi_{\theta}(x) & =\exp \left\{-x^{-\theta}\right\} ; x>0 ; \theta>0, \text { Fréchet distribution, } \\
\Psi_{\theta}(x) & =\exp \left(-(-x)^{\theta}\right) ; x>0, \theta>0 \text {, Weibull distribution. }
\end{aligned}
$$

These one-dimensional extreme values distributions can be unified by the three real-valued parametricdistribution, the so-called generalized extreme values (GEV) model, defined by

$$
\operatorname{GEV}_{(\mu, \sigma, \xi)}(y)=\exp \left\{-\left[1+\xi\left(\frac{y-\mu}{\sigma}\right)\right]_{+}^{(-1 / \xi)}\right\}, \quad \text { if } \xi \neq 0,
$$

where $u_{+}=\max (u, 0)$ and $\{\mu \in \mathbb{R}\},\{\sigma>0\}$, and $\{\xi \in \mathbb{R}\}$ are, respectively, the parameters of location.

Notice that instead of the generalized type of marginal, it is not restrictive to consider any of the three types given by the relation (10). Indeed, these three models are linked each to other by the following functional transformations, even in spatial context:

$$
Y \sim \Phi_{\theta} \Leftrightarrow \ln Y^{\theta} \sim \Lambda \Leftrightarrow \frac{-1}{Y} \sim \Psi_{\theta} \Leftrightarrow Z=\mu(x)+\frac{\sigma(x)}{\xi(x)}\left[Y^{\xi(x)}-1\right] \sim \operatorname{GEV}_{(\sigma(x), \mu(x), \xi(x))}
$$

2.3. An Overview of the Value at Risk. In actuarial and financial sciences, the value at risk (VaR) quantifies numerically the size of the loss for which there is a low probability of being exceeded. It is characterized by the confidence level, the time horizon chosen, and the distribution of profit or loss.
Let $X_{1}, \ldots, X_{n}$ be $n$ random independent variables, identically distributed with common distribution function $F$. A simple way to study the behavior of extreme events is to consider the random variable $M_{n}=\max \left(X_{1}, \ldots, X_{n}\right)$ representing the largest observed loss out of the $n$ observed losses $X_{1}, \ldots, X_{n}$ (we agree that the loss is a positive number). So, for all realization $x$,

$$
P\left(M_{n} \leq x\right)=P\left(\bigcap_{i=1}^{n}\left\{X_{i} \leq x\right\}\right)=F(x, \ldots, x)=C\left\{F_{1}(x), \ldots,\left(F_{1}(x)\right\} .\right.
$$




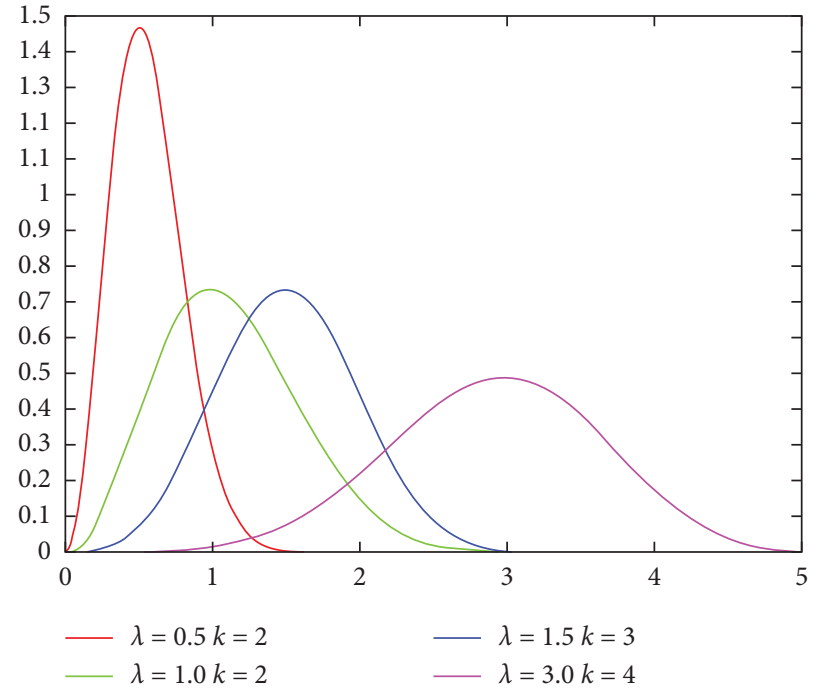

(a)

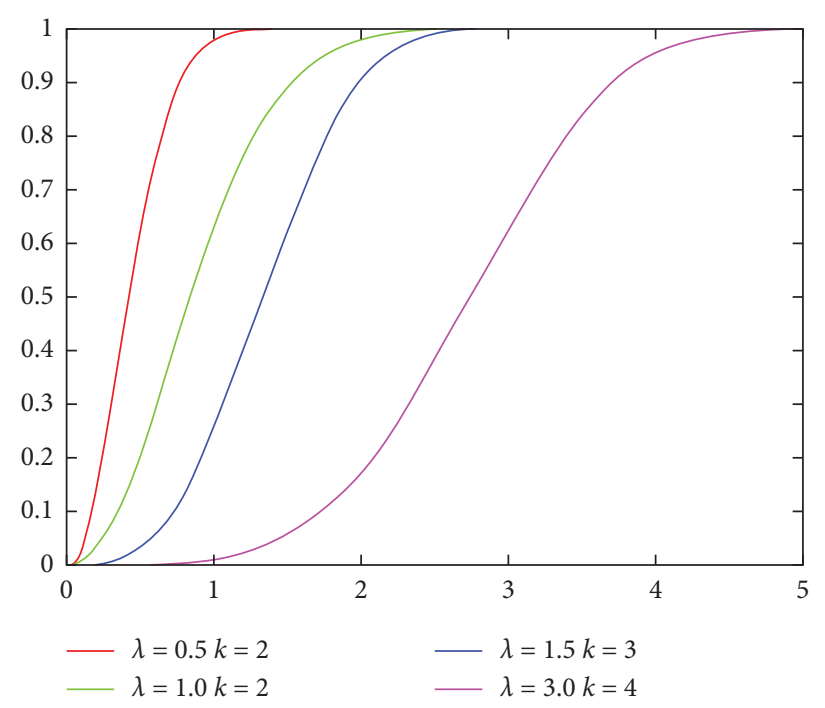

(b)

Figure 1: Density function (a) and distribution function (b) of Weibull.

The value at risk $(\mathrm{VaR})$ is a risk measure mainly used to measure the large portfolio market risk. We call value at risk with a confidence level $\alpha \in$ ]0;1 [ the lower $\alpha$-quantile:

$$
\operatorname{VaR}(X ; \alpha)=\inf \left\{x, F_{X}(x) \geq \alpha\right\}=F_{X}^{-1}(\alpha),
$$

where $F_{X}^{-1}$ is the right continuous inverse of $F_{X}$.

Loyara and Barro [14] showed that the VaR is also intrinsically linked in its expression to this function, making it possible to bridge the copula function with the VaR. More generally, in multivariate studies, for a random vector $X$ satisfying the regularity conditions, one defines the multidimensional VaR at probability level $\alpha$ by

$$
\operatorname{VaR}_{\alpha}(X)=\mathbb{E}[X \mid X \in \partial L(\alpha)],
$$

where $\partial L(\alpha)$ is the boundary of the $\alpha$ - level set of $F_{t}$, the univariate component of the vector.

The three following sections provide the main results of our study.

\section{Portfolio Losses and Default Time Modeling}

Note that in this study, the modeled portfolio contains assets with credit risk. These companies are assigned to one of the industry sectors according to a certain attribute. Sector is further divided into two subsectors according to an attribute. The default times of these companies are denoted as $\tau_{i}$ and are modeled using Weibull law for the parameter $\theta=0$. The default time is modeled as a downtime, with a distribution function

$$
F(t)=P(\tau \leq t)=1-S(t)
$$

where $t \geq 0$ and $S(t)$ is a survival function used to calculate the probability that a time default $\tau$ occurs beyond a certain time interval $t$. The hazard rate $h_{t}$ is defined by $h_{(k, \lambda)}(t)=(f(t) / 1-F(t))$.

$$
h_{(k, \lambda)}(t)=\frac{k}{\lambda}\left(\frac{t}{\lambda}\right)^{k-1}
$$

is increasing $(k \geq 1)$. The default time for an element $i$ of the portfolio is defined as the first time where the expression $\exp \left(-\int_{0}^{t} h_{i}(s) \mathrm{d} s\right)$ falls below the stochastic trigger threshold represented by the variable $U_{i}$.

$$
\tau_{i}=\inf \left\{t: \exp \left\{-\int_{0}^{t} h_{i}(s) \mathrm{d} s\right\} \leq U_{i}\right\}=S^{-1}\left(U_{i}\right)=F^{-1}\left(1-U_{i}\right)
$$

where $U_{i}$ is uniformly distributed over $[0 ; 1]$. In what follows, by coupling Weibull marginal distribution with Gumbel one which is also an extremal copula, we obtain this result.
Proposition 1. Let $C$ be a Gumbel copula which models the dependence of the default times of the assets of a portfolio distributed marginally by the Weibull law. Then, the associated distribution function is 


$$
F\left(t_{1}, \ldots, t_{n}\right)=\exp \left(-\left[\sum_{i=1}^{n}\left\{-\ln \left(1-\exp \left\{-\left(\frac{t_{i}}{\lambda_{i}}\right)^{k_{i}}\right\}\right)\right\}^{\theta}\right]^{1 / \theta}\right)
$$

for all $\left(t_{1}, \ldots, t_{n}\right) \in \mathbb{R}^{n}$ where $\theta$ is the parameter of Gumbel copula $(\theta \geq 1), \lambda_{i}$ and $k_{i}$ are the parameters of Weibull law, and $n$ is the number of the elements of the portfolio.

Proof. According to the relation (1), for all $\left(t_{1}, \ldots, t_{n}\right) \in \mathbb{R}^{n}$, it follows that

$$
F\left(t_{1}, \ldots, t_{n}\right)=C\left(F\left(t_{1}\right), \ldots, F\left(t_{n}\right)\right)
$$

where

$$
C\left(u_{1}, \ldots, u_{n}\right)=\exp \left(-\left[\sum_{1=i}^{n}\left(-\ln \left(u_{i}\right)\right)^{\theta}\right]^{(1 / \theta)}\right) .
$$

So, it follows that

$$
F\left(t_{1}, \ldots, t_{n}\right)=\exp \left(-\left[\sum_{i=1}^{n}\left\{-\ln \left(1-\exp \left\{-\left(\frac{t_{i}}{\lambda_{i}}\right)^{k_{i}}\right\}\right)\right\}^{\theta}\right]^{(1 / \theta)}\right)
$$

While dealing with the distribution of losses of the portfolio, notice that by defining an indicator function $I_{i}(t)=1_{\left\{\tau_{i}<t\right\}}$ at time $t$ and an individual loss $L_{i}^{*}$, the cumulative loss of the portfolio is linked such that

$$
L^{*}(t)=\sum_{i=1}^{n} L_{i}^{*} I_{i}(t)
$$

where for $i=1, \ldots, n$,

$$
L_{i}^{*}=\left(1-R_{c i}\right) A_{i},
$$

where $A_{i}$ is the nominal value of element $i$ of the portfolio and $R_{c i}$ is its recovery rate.

Note that for the distribution of portfolio losses, only the copula function of $\tau_{i}$ is relevant. So, we have the following proposition.
Corollary 1. The copula of losses is given, for all $u_{i} \in[0,1]$ for $i=1, \ldots, n$.

$$
C\left(u_{1}, \ldots, u_{n}\right)=\exp \left(-\left[\sum_{i=1}^{n} \tilde{u}^{\theta}\right]^{(1 / \theta)}\right) .
$$

where $\widetilde{u}_{i}=-\ln \left(u_{i}\right)$ with $\theta \geq 1$.

Proof. With the aim to construct a class of Archimedean copulas belonging to a given domain of attraction, Capéràa et al. [6] have introduced, in bivariate context, the family of Archimax copulas by combining the extreme values and Archimedean copula families into a single class. A member of this class with generator $\varphi$ has the representation, for all $(u, v) \in[0,1]^{2}$,

$$
C_{\phi, A}(u, v)=\phi^{-1}\left(\min \left[\phi(0),(\phi(u)+\phi(v)) A\left(\frac{\phi(u)}{\phi(u)+\phi(v)}\right)\right]\right)
$$

where $A$ is a Pickands dependence function mapping the bivariate unit simplex $S_{2}$ to $[(1 / 2), 1]$, verifying: $\max (t ; 1-t) \leq A(t) \leq 1$, with $S_{n}$ being defined by $S_{n}=$ $\left\{x \in \mathbb{R}_{+}^{n} ;\|x\|_{1}=\sum_{i=1}^{n} x_{i}=1\right\}$.

By noting that only Gumbel copula models the default time, the parametric Archimedean generator is given by $\varphi_{t}(x)=-\ln x$. So, relation (1) allows to obtain

$$
C_{\phi, A}(u, v)=\phi^{-1}\left(\left[(\phi(u)+\phi(v)) A\left(\frac{\phi(u)}{\phi(u)+\phi(v)}\right)\right]\right) .
$$

It comes that, in two dimensional study, one has the Gumbel model:

$$
C(u, v)=\exp \left(-\left[\sum_{i=1}^{n}[-\ln (u)]^{\theta}+[-\ln (v)]^{\theta}\right]^{(1 / \theta)}\right),
$$


which gives by extending to $n$-dimensional cases:

$$
C\left(u_{1}, \ldots, u_{n}\right)=\exp \left(-\left[\sum_{i=1}^{n}\left[-\ln \left(u_{i}\right)\right]^{\theta}\right]^{(1 / \theta)}\right)=\exp \left(-\left[\sum_{i=1}^{n} \tilde{u}^{\theta}\right]^{(1 / \theta)}\right)
$$

where $\tilde{u}_{i}=-\ln \left(u_{i}\right)$ with $\theta \geq 1$.

\section{Nested Archimedean Copulas and Losses}

The class of Archimedean copulas founded a wide range of applications for a number of properties like their computational tractability and their flexibility to model dependencies between random variables, their exchangeability due to the analytic symmetry given by the generator, and the quadrant dependence of the underlying multivariate random vectors see [1] and [15]. However, in multivariate analysis applied in portfolio management, for example, we need nonexchangeable models. To face this drawback, some authors use Laplace transforms to derive more flexible extension of this family of copulas by nesting of generators allowing different degrees of positive dependence in bivariate margins. Denote an $m$-dimensional construction defined interactively for all $\left(u_{1}, \ldots, u_{m}\right) \in[0,1]^{m}$ by

$$
C_{\psi}\left(u_{1}, \ldots, u_{m}\right)=\psi_{0}\left[\psi_{0}^{-1}\left(u_{1}\right)+\psi_{1}^{-1}\left(C_{\Psi_{1}}\left(u_{1}, \ldots, u_{m}\right)\right)\right] .
$$

The inverse $\psi_{0}^{-1}$ of the generator is completely monotone, i.e., for all $t \geq 0$ and $\forall k \geq 0,(-1)^{k}\left(\psi^{-1}\right)^{(k)}(t) \geq 0$ (see [8]).

We present a model based on nested Archimedean copulas (NACs) able to capture hierarchically the structures of dependence between losses in a credit portfolio. The dependence between the losses of all the companies in the same subsector is described by an internal copula $C_{i j}$ of generator $\Phi_{i j}$, and the two subsectors of each sector are then coupled via a copula $C_{i}$ of generator $\Phi_{i}$ and the sectors are then coupled via a copula $C$ of generator $\Phi_{0}$. The companies in a credit portfolio have been classified according to an attribute which is the sector of activity of the industry. Each sector of activity is divided into two subsectors. It yields the following result under the two following assumptions.

Hypothesis 1. Suppose the dependence between the losses of all the companies in the same subsector is described by an internal copula $C_{l i}$ with generator $\Phi_{l i}$; the subsectors are then coupled via a copula $C_{i}$ with generator $\Phi_{l}$ and the sectors via a copula $C$ with generator $\Phi_{0}$. Suppose that the copulas $C_{l i}, C_{l}$, and $C$ are Gumbel copulas.

Hypothesis 2. Suppose the dependence between the losses of all the companies in the same subsector is described by an internal copula $C_{l i}$ with generator $\Phi_{l i}$; the subsectors are then coupled via a copula $C_{i}$ with generator $\Phi_{l}$ and the sectors via a copula $C$ with generator $\Phi_{0}$.

Proposition 2. Let us assume that the dependence between the losses of all the companies in the same subsector is described by an internal copula $C_{l i}$ with generator $\Phi_{l i}$; the subsectors are then coupled via a copula $C_{l}$ with generator $\Phi_{l}$ and the sectors via a copula $C$ with generator $\Phi_{0}$. Then, the partially nested Archimedean copula $C$ is

$$
C(u)=\Phi_{0}^{-1}\left\{\sum_{k=1}^{s} \Phi_{0}\left(\Phi_{l}^{-1}\left[\sum_{i=1}^{2} \Phi_{l}\left(\Phi_{l i}^{-1}\left\{\sum_{j=1}^{d_{l i}} \Phi_{k i}\left(u_{j}^{l i}\right)\right\}\right)\right]\right\}\right.
$$

where $u=\left(u_{1}^{11}, \ldots, u_{d_{s 2}}^{s 2}\right), s$ is the number of business sectors, and $d_{l i}$ is the number of businesses in subsector $i$ of sector $l$, such that

$$
\sum_{i=1}^{2} \sum_{l=1}^{s} d_{l i}=n
$$

with $n$ being the number of companies and $d_{l i}$ being the number of companies belonging to subsector $i$ of sector $l$.

Proof. Let $C_{l}$ and $C_{l i}$ be Archimedean copulas with generators $\Phi_{l}$ and $\Phi_{l i}$, respectively. One has

$$
C_{1}\left(u_{1}\right)=\Phi_{1}^{-1}\left[\Phi_{1}\left(C_{11}\left(u_{1}\right)\right)+\Phi_{1}\left(C_{12}\left(u_{1}\right)\right)\right]
$$

which gives

$$
C_{1}\left(u_{1}\right)=\Phi_{1}^{-1}\left\{\Phi _ { 1 } \left[\Phi_{11}^{-1}\left(\Phi_{11}\left(u_{1}^{11}\right)+\cdots+\Phi_{11}\left(u_{d_{11}}^{11}\right)\right)+\left(\Phi_{1}\left(\Phi_{12}^{-1}\left(\Phi_{12}\left(u_{1}^{12}\right)+\cdots+\Phi_{12}\left(u_{d_{12}}^{12}\right)\right)\right]\right\}\right.\right.
$$

Furthermore, for the first sector, it follows that

$$
C_{1}\left(u_{1}\right)=\Phi_{1}^{-1}\left\{\sum_{i=1}^{2} \Phi_{1}\left(\Phi_{1 i}^{-1}\left[\sum_{j=1}^{d_{1 i}}\left(\Phi_{1 i}\left(u_{j}^{1 i}\right)\right)\right]\right)\right\} \text {. }
$$

which provides, for the second member,

$$
C_{2}\left(u_{2}\right)=\Phi_{2}^{-1}\left\{\sum_{i=1}^{2} \Phi_{2}\left(\Phi_{2 i}^{-1}\left[\sum_{j=1}^{d_{2 i}} \Phi_{2 i}\left(u_{j}^{2 i}\right)\right]\right)\right\} \text {. }
$$


So, more generally, for all $l \in\{1,2, \ldots, s\}$,

$$
C_{l}\left(\left(u_{1}^{l 1}, \ldots, u_{d_{l i}}^{l 2}\right)\right)=\Phi_{l}^{-1}\left(\sum_{i=1}^{2}\left\{\Phi_{l}\left[\Phi_{l i}^{-1}\left(\sum_{j=1}^{d_{l i}} \Phi_{l i}\left(u_{j}^{l i}\right)\right)\right]\right\}\right) \text {. }
$$

$$
C\left(u_{1}^{11}, \ldots, u_{d_{s 2}}^{s 2}\right)=C\left(C_{1}, \ldots, C_{s}\right)=\Phi_{0}^{-1}\left(\sum_{l=1}^{s} \Phi_{0}\left(C_{l}\right)\right)
$$

which gives

Furthermore, the copula $C$ is given by

$$
C\left(u_{1}^{11}, \ldots, u_{d_{s 2}}^{s 2}\right)=\Phi_{0}^{-1}\left(\sum_{l=1}^{s} \Phi_{0}\left\{\Phi_{l}^{-1}\left(\sum_{i=1}^{2} \Phi_{l}\left\{\Phi_{l i}^{-1}\left[\sum_{j=1}^{d_{l i}}\left(\Phi_{l i}\left(u_{j}^{l i}\right)\right)\right]\right\}\right)\right\}\right)
$$

where $s$ is the number of companies sectors $\sum_{i=1}^{2} \sum_{l=1}^{s} d_{l i}=n$, while $n$ represents the number of companies. (i) The dependence of losses on companies in the same sector is given by

Proposition 3. Suppose that the copulas $C_{l i}, C_{l}$, and $C$ are Gumbel copulas. Then,

$$
C_{l}\left(u_{1}^{l 1}, \ldots, u_{d_{l 1}}^{l 1}, u_{1}^{l 2}, \ldots, u_{d_{l 2}}^{l 2}\right)=\exp \left\{-\left[\sum_{i=1}^{2}\left(\sum_{j=1}^{d_{l i}}\left[-\ln \left(u_{j}^{l i}\right)\right]^{\theta_{l i}}\right)^{\left(\theta_{l} / \theta_{l i}\right)}\right]^{\left(1 / \theta_{l}\right)}\right\}
$$

(ii) The dependence of losses on portfolio companies is given by

$$
C\left(C_{1}, \ldots, C_{s}\right)=C\left(u_{1}^{11}, \ldots, u_{d_{s 2}}^{s 2}\right)=\exp \left\{-\left[\sum_{l=1}^{s}\left(-\ln \left[\exp \left\{-\left[\sum_{i=1}^{2}\left(\sum_{j=1}^{d_{l i}}\left[-\ln \left(u_{j}^{l i}\right)\right]^{\theta_{l i}}\right)^{\left(\theta_{l} / \theta_{l i}\right)}\right]^{\left(1 / \theta_{l}\right)}\right\}\right]\right)^{\theta_{o}}\right]^{\left(1 / \theta_{o}\right)}\right\}
$$

The parameters $\theta_{0}, \theta_{l}$, and $\theta_{l i}$ of copulas $C, C_{l}$, and $C_{l j}$ satisfy the condition $\theta_{0}<\theta_{l}<\theta_{l i}$ with $l \in\{1, \ldots, s\}$ and $i \in\{1,2\}$.

$$
C_{l i}\left(u_{1}^{l i}, \ldots, u_{d_{l i}}^{l i}\right)=\exp \left\{-\left(\sum_{j=1}^{d_{l i}}\left[-\ln \left(u_{j}^{l i}\right)\right]^{\theta_{l i}}\right)^{1 / \theta_{l i}}\right\}
$$

Proof

Therefore, one has

(i) Let $C_{l i}$ and $C_{l}$ be Gumbel copulas of parameters $\theta_{l i}$ and

$\theta_{l}$, respectively; one has

$$
C_{l}\left(C_{l 1}, C_{l 2}\right)=C_{l}\left(u_{1}^{l 1}, \ldots, u_{d_{l 1}}^{l 1}, u_{1}^{l 2}, \ldots, u_{d_{l 2}}^{l 2}\right)=\exp \left\{-\left[\sum_{i=1}^{2}\left(\sum_{j=1}^{d_{l i}}\left[-\ln \left(u_{j}^{l i}\right)\right]^{\theta_{l i}}\right)^{\left(\theta_{l} / \theta_{l i}\right)}\right]^{\left(1 / \theta_{l}\right)}\right\}
$$

Finally, it follows that 


$$
C_{l}\left(u_{1}^{l 1}, \ldots, u_{d_{l 1}}^{l 1}, u_{1}^{l 2}, \ldots, u_{d_{l 2}}^{l 2}\right)=\exp \left\{-\left[\sum_{i=1}^{2}\left(\sum_{j=1}^{d_{l i}}\left[-\ln \left(u_{j}^{l i}\right)\right]^{\theta_{l i}}\right)^{\left(\theta_{l} / \theta_{l i}\right)}\right]^{\left(1 / \theta_{l}\right)}\right\}
$$

(ii) Let $C_{l i}$ and $C_{l}$ be Gumbel copulas of parameters $\theta_{l i}$ and $\theta_{l}$, respectively; one has

$$
C\left(C_{1}, \ldots, C_{s}\right)=C\left(u_{1}^{11}, \ldots, u_{d_{s 2}}^{s 2}\right)=\exp \left\{-\left[\sum_{l=1}^{s}\left(-\ln \left[\exp \left\{-\left[\sum_{i=1}^{2}\left(\sum_{j=1}^{d_{l i}}\left[-\ln \left(u_{j}^{l i}\right)\right]^{\theta_{\theta_{i}}}\right)^{\left(\theta_{l} / \theta_{i i}\right)}\right]^{\left(1 / \theta_{l}\right)}\right\}\right]\right)^{\theta_{o}}\right]^{\left(1 / \theta_{o}\right)}\right\}
$$

The parameters $\theta_{0}, \theta_{l}$, and $\theta_{l i}$ of copulas $C, C_{l}$, and $C_{l i}$ satisfy the condition $\theta_{0}<\theta_{l}<\theta_{l i}$ with $l \in\{1, \ldots, s\}$ and $i \in\{1,2\}$ (see [15]).

\section{Sampling the Maximum Values at Risk in a Portfolio}

5.1. On the Maximum of the VaR in Each Subsector. Here we determine the maximum of the value at risk in each subsector.

Proposition 4. Suppose the dependence between the losses of all the companies in the same subsector is described by a
Gumbel copula $C_{l i}$ of parameter $\theta_{l i}$ and that the default times are identically distributed by the law of Weibull. Then, the maximum of value at risk in each subsector is given by

$$
\operatorname{VaR}_{l i}^{\max }(\alpha)=\lambda\left[-\ln \left\{1-\exp \left(\frac{\ln (\alpha)}{d_{l i}^{\left(1 / \theta_{l i}\right)}}\right)\right\}\right]^{(1 / k)},
$$

where $\lambda$ and $k$ are the parameters of Weibull law and $d_{l i}$ is the number of elements in the subsector li.

Proof. According to the relation (1), one has

$$
F\left(t_{1}, \ldots, t_{n}\right)=C_{F}\left(F_{1}\left(t_{1}\right), \ldots, F_{n}\left(t_{n}\right)\right), \quad \text { with } F_{i}\left(t_{i}\right)=1-\exp \left\{-\left(\frac{t_{i}}{\lambda}\right)^{k}\right\}
$$

where if $\left(t_{1}^{l i}, \ldots, t_{d_{l i}}^{l i}\right)=(t, \ldots, t)$,

$$
F_{l i}\left(t_{1}^{l i}, \ldots, t_{d_{l i}}^{l i}\right)=C_{l i}\left(F_{1}^{l i}\left(t_{1}^{l i}\right), \ldots, F_{d_{l i}}^{l i}\left(t_{d_{l i}}^{l i}\right)\right)=\exp \left(d_{l i}^{\left(1 / \theta_{l i}\right)} \ln \left[1-\exp \left\{-\left(\frac{t}{\lambda}\right)^{k}\right\}\right]\right)
$$

One has $\operatorname{VaR}_{l i}^{\max }(\alpha)=F_{l i}^{-1}(\alpha)$, where

$$
F_{l i}\left(t_{1}^{l i}, \ldots, t_{d_{l i}}^{l i}\right)=\alpha \text {. }
$$

That is,

$$
\exp \left(d_{l i}^{\left(1 / \theta_{l i}\right)} \ln \left[1-\exp \left\{-\left(\frac{t}{\lambda}\right)^{k}\right\}\right]\right)=\alpha .
$$

Equivalently, it follows that

$$
d_{l i}^{\left(1 / \theta_{l i}\right)} \ln \left[1-\exp \left\{-\left(\frac{t}{\lambda}\right)^{k}\right\}\right]=\ln (\alpha) .
$$

Thus, it follows that

$$
1-\exp \left\{-\left(\frac{t}{\lambda}\right)^{k}\right\}=\exp \left(\frac{\ln (\alpha)}{d_{l i}^{\left(1 / \theta_{l i}\right)}}\right)
$$

Finally, one has

$$
t=\left(-\lambda^{k} \ln \left[1-\exp \left(\frac{\ln (\alpha)}{d_{l i}^{\left(1 / \theta_{l i}\right)}}\right)\right]\right)^{(1 / k)} .
$$

So, one obtains easily

$$
\operatorname{VaR}_{l i}^{\max }(\alpha)=\lambda\left\{-\ln \left[1-\exp \left(\frac{\ln (\alpha)}{d_{l i}^{\left(1 / \theta_{l i}\right)}}\right)\right]\right\}^{(1 / k)},
$$


which proved the result as disserted.

Figure 2 is a graphic representation of $\mathrm{VaR}_{l i}^{\max }$ where some parameters are fixed.

The graph shows that the maximum of the value at risk is an increasing function of alpha for the parameters which are fixed. With other parameters, we obtain the same property.

5.2. Computation of the Maximum Potential Loss in Each Sector. In this subsection, we determine the maximum of value at risk in each sector.
Proposition 5. Let us presume that the dependence between the losses of all the companies in the same subsector is described by a Gumbel copula $C_{l i}$ with parameter $\theta_{l i}$; the two subsectors of each sector are then coupled via a copula $C_{l}$ with parameter $\theta_{l}$. And if the default times are identically distributed by Weibull law, then the maximum of value at risk in each sector is

$$
\operatorname{VaR}_{l}^{\max }(\alpha)=\lambda\left[-\ln \left[1-\exp \left\{\ln (\alpha)\left(d_{l 1}^{\left(\theta_{l} / \theta_{l 1}\right)}+d_{l 2}^{\left(\theta_{l} / \theta_{l 2}\right)}\right)^{\left((-1) / \theta_{l}\right)}\right\}\right]\right]^{(1 / k)}
$$

where $\lambda$ and $k(k \geq 1)$ are the parameters of Weibull distribution and $d_{l 1}$ and $d_{l 1}$ are, respectively, the number of companies of subsectors $l 1$ and $l 2$.
Proof. According to the canonical parametric relation (1), $F\left(t_{1}, \ldots, t_{n}\right)=C\left(F_{1}\left(t_{1}\right), \ldots, F_{n}\left(t_{n}\right)\right) \quad$ with $\quad F_{i}\left(t_{i}\right)=1-$ $e^{-\left(t_{i} / \lambda\right) k}$. So, it follows that

$$
C_{l}\left(u_{1}^{l 1}, \ldots, u_{d_{l 1}}^{l 1}, u_{1}^{l 2}, \ldots, u_{d_{l 2}}^{l 2}\right)=\exp \left\{-\left[\sum_{i=1}^{2}\left(\sum_{j=1}^{d_{l i}}\left[-\ln \left(u_{j}^{l i}\right)\right]^{\theta_{l i}}\right)^{\left(\theta_{l} / \theta_{l i}\right)}\right]^{\left(1 / \theta_{l}\right)}\right\}
$$
has

Moreover, if $\left(t_{1}^{l 1}, \ldots, t_{d_{l 1}}^{l 1}, t_{1}^{l 2}, \ldots, t_{d_{l 2}}^{l 2}\right)=(t, \ldots, t)$, one

$$
F_{l}\left(t_{1}^{l 1}, \ldots, t_{d_{l 1}}^{l 1}, t_{1}^{l 2}, \ldots, t_{d_{l 2}}^{l 2}\right)=\exp \left[-\left(d_{l 1}\left[-\ln \left(1-\exp \left(-\left(\frac{1}{\lambda} t\right)^{k}\right)\right)\right]^{\theta_{l 1}}\right)^{\left(\theta_{l} / \theta_{l 1}\right)}+\left(d_{l 2}\left[-\ln \left(1-\exp \left(-\left(\frac{1}{\lambda} t\right)^{k}\right)\right]^{\theta_{l 2}}\right)^{\left(\theta_{l} / \theta_{l 2}\right)}\right]^{\left(1 / \theta_{l}\right)}\right.
$$

which is equivalent to

$$
\left.F_{l}\left(t_{1}^{l 1}, \ldots, t_{d_{l 1}}^{l 1}, t_{1}^{l 2}, \ldots, t_{d_{l 2}}^{l 2}\right)=\exp \left[-\left\{d_{l 1}^{\left(\theta_{l} / \theta_{l 1}\right)}\left[-\ln \left\{1-\exp \left(-\left(\frac{1}{\lambda} t\right)^{k}\right)\right\}\right]^{\theta_{l}}+d_{l 2}^{\left(\theta_{l} / \theta_{l 2}\right)}\left[-\ln \left(1-\exp \left\{-\left(\frac{t}{\lambda}\right)^{k}\right\}\right)\right]^{\theta}\right\}\right]^{\left(1 / \theta_{l}\right)}\right]
$$




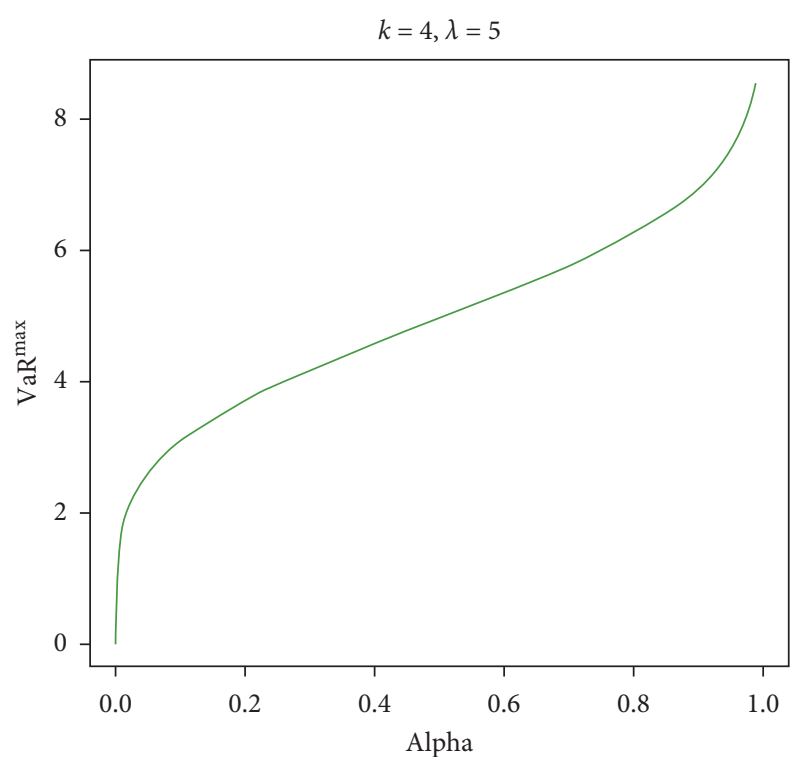

Figure 2: Maximum of the value at risk as a function of confidence level $\alpha$ for $d_{l i}=20, \theta_{l i}=8$ for all $l \in\{1, \ldots, s\}$ and $i \in\{1,2\}$ in a subsector.

which provides

which means that

$\operatorname{VaR}_{l}(\alpha)=F^{-1}(\alpha), \quad$ and $F_{l}\left(t_{1}^{l 1}, \ldots, t_{d_{l 1}}^{l 1}, \ldots, t_{d_{l 2}}^{l 2}\right)=\alpha$

$$
\exp \left\{-\left(d_{l 1}^{\left(\theta_{l} / \theta_{l 1}\right)}\left[-\ln \left(1-\exp \left\{-\left(\frac{t}{\lambda}\right)^{k}\right\}\right)\right]^{\theta_{l}}+d_{l 2}^{\left(\theta_{l} / \theta_{l 2}\right)}\left[-\ln \left(1-\exp \left\{-\left(\frac{t}{\lambda}\right)^{k}\right\}\right)\right]^{\theta_{l}}\right)^{\left(1 / \theta_{l}\right)}\right\}=\alpha
$$

Therefore, it follows that

$$
\left[-\ln \left(1-\exp \left\{-\left(\frac{t}{\lambda}\right)^{k}\right\}\right)\right]^{\theta_{l}}=\frac{[-\ln (\alpha)]^{\theta_{l}}}{d_{l 1}^{\left(\theta_{l} / \theta_{l 1}\right)}+d_{l 2}^{\left(\theta_{l} / \theta_{l 2}\right)}}
$$

$$
t=\left[-\lambda^{k} \ln \left\{1-\exp \left(\frac{\ln (\alpha)}{\left(d_{l 1}^{\left(\theta_{l} / \theta_{l 1}\right)}+d_{l 2}^{\left(\theta_{l} / \theta_{l 2}\right)}\right)^{\left(1 / \theta_{l}\right)}}\right)\right\}\right]^{(1 / k)},
$$

From the above formula, one can have

and finally

$$
\operatorname{VaR}_{l}^{\max }(\alpha)=\lambda\left[-\ln \left[1-\exp \left\{\ln (\alpha)\left(d_{l 1}^{\left(\theta_{l} / \theta_{l 1}\right)}+d_{l 2}^{\left(\theta_{l} / \theta_{l 2}\right)}\right)^{\left(-1 / \theta_{l}\right)}\right\}\right]\right]^{(1 / k)}
$$

So, the result is proved. Figure 3 is a graphic representation of $\mathrm{VaR}_{l}^{\max }$ where some parameters are fixed.

The graph shows that the maximum of the value at risk is an increasing function of alpha for the parameters which are fixed. With other parameters, we obtain the same property.
5.3. On the Maximum of the VaR in the Portfolio. In this section, we compute the maximum of value at risk in the portfolio made up of securities belonging to the different sectors and its variation as a function of $\lambda$ and $k$. 


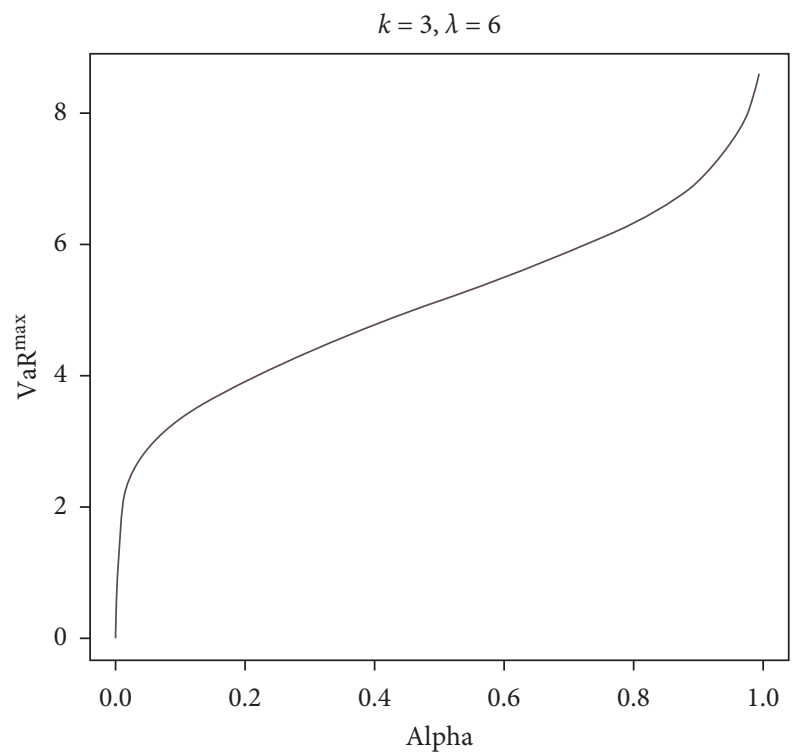

Figure 3: Maximum of the value at risk as a function of confidence level $\alpha$ for $d_{l i}=20, \theta_{l}=5, \theta_{l 1}=8, \theta_{l 2}=7,5$ for all $l \in\{1, \ldots, s\}$ and $i \in\{1,2\}$ in a sector.

Proposition 6. Suppose the dependence between the losses of all the companies in the same subsector is described by a Gumbel copula $C_{l i}$ with parameter $\theta_{l i}$; the two subsectors of each sector are then coupled via a copula $C_{l}$ with parameter $\theta_{l}$ and the sectors are coupled via a copula $C$ with parameter $\theta_{0}$. And if the default times are identically distributed by Weibull law, then the maximum potential loss of the portfolio is

$$
\operatorname{VaR}^{\max }(\alpha)=\lambda\left[-\ln \left(1-\exp \left[\ln (\alpha)\left[\sum_{l=1}^{s}\left(d_{l 1}^{\left(\theta_{l} / \theta_{l 1}\right)}+d_{l 2}^{\left(\theta_{l} / \theta_{l 2}\right)}\right)^{\left(\theta_{0} / \theta_{l}\right)}\right]^{\left(-1 / \theta_{0}\right)}\right]\right)\right]^{(1 / k)}
$$

where $\lambda$ and $k(k \geq 1)$ are the parameters of Weibull distribution with $d_{l 1}$ and $d_{l 1}$ being, respectively, the number of companies of subsectors $l 1$ and $l 2$.

$$
F\left(t_{1}^{11}, \ldots, t_{d_{s 2}}^{s 2}\right)=C\left(F_{1}^{11}\left(t_{1}^{11}\right), \ldots, F_{d_{s 2}}^{s 2}\left(t_{d_{s 2}}^{s 2}\right)\right) \text {. }
$$

So, if $\left(t_{1}^{11}, \ldots, t_{d_{s 2}}^{s 2}\right)=(t, \ldots, t)$, one has

Proof. Using the parametric form of Sklar theorem, it follows that

$$
F\left(t_{1}^{11}, \ldots, t_{d_{s 2}}^{s 2}\right)=\exp \left(-\left[\sum_{l=1}^{s}\left(-\ln \left(\exp \left[-\left[d_{l_{1}}^{\left(\theta_{l} / \theta_{l_{1}}\right)}\left(-\ln \left[1-\exp \left(-\left(\frac{t}{\lambda}\right)^{k}\right)\right]\right)^{\theta_{l}}+d_{l_{2}}^{\left(\theta_{l} / \theta_{l_{2}}\right)}\left(-\ln \left[1-\exp \left(-\left(\frac{t}{\lambda}\right)^{k}\right)\right]\right)^{\theta_{l}}\right]^{\left(1 / \theta_{l}\right)}\right]\right)\right)^{\theta_{0}}\right]^{\left(1 / \theta_{0}\right)}\right]
$$

Since

$$
F\left(t_{1}^{11}, \ldots, t_{d_{s 2}}^{s 2}\right)=\alpha
$$

$$
\left[\sum_{l=1}^{s}\left(-\ln \left(\exp \left[-\left[d_{l_{1}}^{\left(\theta_{l} / \theta_{l_{1}}\right)}\left(-\ln \left[1-\exp \left(-\left(\frac{t}{\lambda}\right)^{k}\right)\right]\right)^{\theta_{l}}+d_{l_{2}}^{\left(\theta_{l} / \theta_{l_{2}}\right)}\left(-\ln \left[1-\exp \left(-\left(\frac{t}{\lambda}\right)^{k}\right)\right]\right)^{\theta_{l}}\right]^{\left(1 / \theta_{l}\right)}\right]\right)\right)^{\theta_{0}}\right]^{\left(1 / \theta_{0}\right)}=-\ln (\alpha)
$$


It follows that

$$
\left.\sum_{l=1}^{s}\left(-\ln \left(\exp \left[-\left[d_{l_{1}}^{\left(\theta_{l} / \theta_{l_{1}}\right)}\left(-\ln \left[1-\exp \left(-\left(\frac{t}{\lambda}\right)^{k}\right)\right]\right)^{\theta_{l}}+d_{l_{2}}^{\left(\theta_{l} / \theta_{l_{2}}\right.}\right)\left(-\ln \left[1-\exp \left(-\left(\frac{t}{\lambda}\right)^{k}\right)\right]\right)^{\theta_{l}}\right]^{\left(1 / \theta_{l}\right)}\right]\right)\right)^{\theta_{0}}=[-\ln (\alpha)]^{\theta_{0}}
$$

Moreover, $\left.\left[\sum_{l=1}^{s}\left(d_{l} 1^{\left(\theta_{l} / \theta_{l_{1}}\right)}+d_{l 2}^{\left(\theta_{l} /\right.} \theta_{l_{2}}\right)\right)^{\left(\theta_{0} / \theta_{l}\right)}\right][-\ln (1-$ $\left.\left.\exp \left(-(t / \lambda)^{k}\right)\right)\right]^{\theta_{0}}=[-\ln (\alpha)]^{\theta_{0}}$. Therefore, it follows that

$$
\left[-\ln \left\{1-\exp \left(-\left(\frac{t}{\lambda}\right)^{k}\right)\right\}\right]^{\theta_{0}}=[-\ln (\alpha)]^{\theta_{0}}\left[\sum_{l=1}^{s}\left(d_{l 1}^{\left(\theta_{l} / \theta_{l_{1}}\right)}+d_{l 2}^{\left(\theta_{l} / \theta_{l_{2}}\right)}\right)^{\left(\theta_{0} / \theta_{l}\right)}\right]^{-1}
$$

We obtain

$$
-\ln \left\{1-\exp \left(-\left(\frac{t}{\lambda}\right)^{k}\right)\right\}=[-\ln (\alpha)]\left[\sum_{l=1}^{s}\left(d_{l 1}^{\left(\theta_{l} / \theta_{l_{1}}\right)}+d_{l 2}^{\left(\theta_{l} / \theta_{l_{2}}\right)}\right)^{\left(\theta_{0} / \theta_{l}\right)}\right]^{\left(-1 / \theta_{0}\right)} .
$$

It follows that

$$
\exp \left[-\left(\frac{t}{\lambda}\right)^{k}\right]=1-\exp \left\{\ln (\alpha)\left[\sum_{l=1}^{s}\left(d_{l 1}^{\left(\theta_{l} / \theta_{l_{1}}\right)}+d_{l 2}^{\left(\theta_{l} / \theta_{l_{2}}\right)}\right)^{\left(\theta_{0} / \theta_{l}\right)}\right]^{\left(-1 / \theta_{0}\right)}\right\}
$$

Finally, we have

$$
t=\lambda\left[-\ln \left(1-\exp \left[\ln (\alpha)\left[\sum_{l=1}^{s}\left(d_{l 1}^{\left(\theta_{l} / \theta_{l_{1}}\right)}+d_{l 2}^{\left(\theta_{l} / \theta_{l_{2}}\right)}\right)^{\left(\theta_{0} / \theta_{l}\right)}\right]^{\left(-1 / \theta_{0}\right)}\right]\right)\right]^{(1 / k)}
$$

So,

$$
\operatorname{VaR}^{\max }(\alpha)=\lambda\left[-\ln \left(1-\exp \left[\ln (\alpha)\left[\sum_{l=1}^{s}\left(d_{l 1}^{\left(\theta_{l} / \theta_{l_{1}}\right)}+d_{l 2}^{\left(\theta_{l} / \theta_{l_{2}}\right)}\right)^{\left(\theta_{0} / \theta_{l}\right)}\right]^{\left(-1 / \theta_{0}\right)}\right]\right)\right]^{(1 / k)}
$$




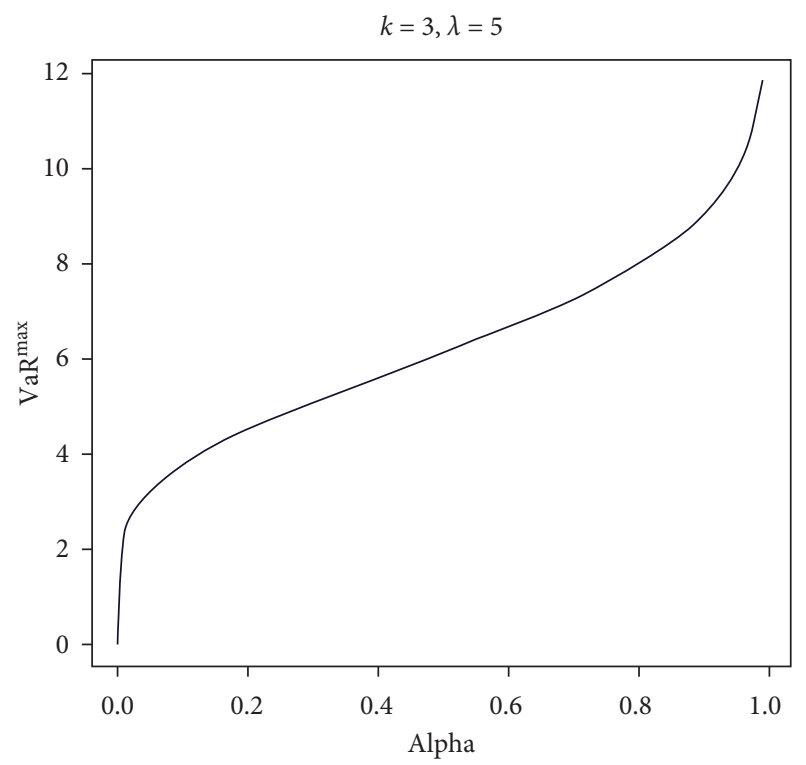

Figure 4: Maximum of the value at risk as a function of confidence level $\alpha$ for $d_{l i}=20, \theta_{1}=5, \theta_{2}=6, \theta_{3}=7, \theta_{11}=8, \theta_{12}=7,5, \theta_{21}=8$, $\theta_{22}=9, \theta_{31}=8,5, \theta_{32}=9,5$ for all $l \in\{1, \ldots, s\}$ and $i \in\{1,2\}$ in the portfolio made up of 3 sectors.

Thus, the result is proved as disserted.

$\mathrm{VaR}^{\max }$ depends on $\lambda, k$ and on correlations between and decreasing otherwise. sectors, subsectors, and companies in the same sector. The following theorem is a key result of our study.

Proof

Proposition 7. The maximum potential loss of the portfolio satisfies the following properties:

(i) From the previous results, one can write that

(i) $V a R^{\max }$ is an increasing function of $\lambda$.

(ii) It is an increasing function of $k$ if

$$
\left[\sum_{l=1}^{s}\left(d_{l 1}^{\left(\theta_{l} / \theta_{l_{1}}\right)}+d_{l 2}^{\left(\theta_{l} / \theta_{l_{2}}\right)}\right)^{\left(\theta_{0} / \theta_{l}\right)}\right]^{\left(1 / \theta_{0}\right)} \leq \frac{\ln (\alpha)}{\ln \left(1-e^{-1}\right)}
$$

$$
\operatorname{VaR}^{\max }(\alpha)=\lambda\left[-\ln \left(1-\exp \left[\ln (\alpha)\left[\sum_{l=1}^{s}\left(d_{l 1}^{\left(\theta_{l} / \theta_{l_{1}}\right)}+d_{l 2}^{\left(\theta_{l} / \theta_{l_{2}}\right)}\right)^{\left(\theta_{0} / \theta_{l}\right)}\right]^{\left(11 / \theta_{0}\right)}\right]\right)\right]^{(1 / k)}
$$

So, we obtain easily

$$
\left.\left.\left.\frac{\partial V a R^{\max }(\alpha)}{\partial \lambda}=\left[-\ln \left(1-\exp \left[\ln (\alpha)\left[\sum_{l=1}^{s}\left(d_{l 1}^{\frac{\theta_{l}}{\theta_{l 1}}}+\frac{\theta_{l 2}}{\theta_{l 2}}\right)^{\frac{\theta_{0}}{\theta_{l}}}\right]\right]\right)\right]\right]^{\frac{-1}{\theta_{0}}}\right]\right]^{\frac{1}{k}}>
$$


(ii) Let us denote $f(k)=x^{(1 / k)}$, a function $f$ defined on $[1,+\infty$ [ for $x>0$. It is obvious that $f$ is increasing in $k$ if $0<x \leq 1$ and decreasing if $x>1$. So, one obtains the result as disserted.

Figure 4 is a graphic representation of $\mathrm{VaR}^{\max }$ where some parameters are fixed.

The graph shows that maximum of the value at risk of the portfolio is an increasing function of alpha for the parameters which are fixed. This result is demonstrated and generalized by the previous proposition.

\section{Conclusion}

In this study, we contribute to the modeling of the dependence of losses of a large portfolio. Mainly, using a negative Weibulldistributed law and a family of nested Archimedean copulas, we estimate the highest value of the VaR. The use of the threelevel nested Archimedean copula with Gumbel's copulas provides interesting results on the maximum of value at risk of the credit portfolio divided into subsectors and sectors when the default time follows Weibull law. It has been shown that this maximum of value at risk of the portfolio is an increasing function of $\lambda$. But it is an increasing or decreasing of $k$ according to the conditions given by Proposition 7 .

\section{Data Availability}

This is a theoretical study. No data were used to support this study.

\section{Conflicts of Interest}

The authors declare that they have no conflicts of interest.

\section{References}

[1] R. B. Nelsen, "An introduction to copulas," in Lecture Notes in Statistics, Vol. 139, Springer, Berlin, Germany, 2006.

[2] C. Savu and M. Trede, "Hierarchical archimedean copula," Quantitative Finance, vol. 10, no. 3, pp. 295-304, 2009.

[3] J. M. Hofert, "Sampling nested archimedean copulas with applications to CDO pricing," Open Access Repositorium der Universität Ulm, vol. 2.

[4] A. J. McNeil, "Sampling nested archimedean copulas," Journal of Statistical Computation and Simulation, vol. 78, no. 6, pp. 561-581, 2008.

[5] P. Schonbucher, Credit Derivatives Pricing Models, Wiley, Chichester, UK, 2003.

[6] P. Capéraà, A.-L. Fougères, and C. Genest, "Bivariate distributions with given extreme value attractor," Journal of Multivariate Analysis, vol. 72, no. 1, pp. 30-49, 2000.

[7] U. Cherubini, E. Luciano, and W. Vecchiato, "Copula methods in finance," Wiley Series in Financial Engineering, Wiley, Chichester, UK, 2004.

[8] M. Hofert and M. Scherer, "CDO pricing with nested archimedean copulas," Quantitative Finance, vol. 11, no. 5, pp. 775-787, 2011.

[9] R. Veysseyre, Statistique et Probabilité Pour L'ingénieur, Dunod, Paris, France, 2e edition, 2006.
[10] R. Tyrrell Rockafellar and S. Uryasev, "Optimization of conditional value-at-risk," Journal of Risk, vol. 54, pp. 411435, 2000.

[11] T. Roncalli, Gestion des Risques Multiples, Collection Finance, 2e edition, 2009.

[12] L. de Weibull, "Dans wikipedia," 2021, https://fr.wikipedia. org/wiki/Loi_de_Weibull.

[13] P. Embrechts, K. Claudia, and M. Thomas, "Modélisation d'événements extrêmes," L'assurance et la Finance, Spring Verlag, Berlin, Germany, 1997.

[14] V. Y. B. Loyara and D. Barro, "Value-at-risk modeling with conditional copulas in euclidean space framework," European Journal of Pure and Applied Mathematics, vol. 12, no. 1, 2019.

[15] O. Okhrin, Y. Okhrin, and W. Schmid, "Properties of hierarchical archimedean copulas," Statistics and Risk Modeling, vol. 30 , no. $1,2013$. 